\title{
A technology-enabled adherence enhancement system for people with bipolar disorder: results from a feasibility and patient acceptance analysis
}

This article was published in the following Dove Press journal:

Patient Preference and Adherence

8 June 2015

Number of times this article has been viewed

\author{
Martha Sajatovic' \\ Michael S Davis ${ }^{2}$ \\ Kristin A Cassidy ${ }^{3}$ \\ Joseph Nestor ${ }^{2}$ \\ Johnny Sams ${ }^{3}$ \\ Edna Fuentes-Casiano ${ }^{3}$ \\ 'Department of Psychiatry and \\ Neurological and Behavioral \\ Outcomes Center, Case Western \\ Reserve University School of Medicine \\ and University Hospitals Case \\ Medical Center, Cleveland, OH, USA; \\ ${ }^{2}$ MedicaSafe, New York, NY, USA; \\ ${ }^{3}$ Department of Psychiatry, Case \\ Western Reserve University School \\ of Medicine, University Hospitals \\ Case Medical Center, Cleveland, \\ $\mathrm{OH}, \mathrm{USA}$
}

Objective: As poor medication adherence is common in bipolar disorder (BD), technologyassisted approaches may help to monitor and enhance adherence. This study evaluated preliminary feasibility, patient satisfaction and effects on adherence, BD knowledge, and BD symptoms associated with the use of a multicomponent technology-assisted adherence enhancement system.

Methods: This prospective study tested the system in five BD patients over a 15-day period. System components included: 1) an automated pill cap with remote monitoring sensor; 2) a multimedia adherence enhancement program; and 3) a treatment incentive program. This study evaluated system usability, patient satisfaction and effects on adherence (Morisky scale), knowledge (treatment knowledge test [TKT]), and symptoms (internal state scale [ISS]).

Results: Mean age of the sample was 62 years, 4/5 (80\%) Caucasian, and 4/5 (80\%) single/divorced or widowed. Most participants $(4 / 5,80 \%)$ were on a single BD medication. Participants had BD for an average of 21 years. Challenges included attaching the pill sensor to standard pharmacy bottles for individuals using very large pill containers or those with multiday pill boxes. Three of five (60\%) individuals completed the full 15-day period. Usability scores were high overall. Mean Morisky scores improved. Means on all four subscales of the ISS were all in the direction of improvement. On the TKT, there was a $40 \%$ increase in mean scores.

Conclusion: A multicomponent technology-assisted BD adherence enhancement system is feasible. Challenges include accommodating multiple types of pill containers and monitoring multiple drugs simultaneously. The system can also generate adherence information that is potentially useful for treatment planning.

Keywords: adherence, compliance, bipolar disorder, manic depressive disorder, mood stabilizers

\section{Introduction}

Bipolar disorder (BD) is a chronic mental illness associated with reduced individual quality of life, devastating effects on families and children, and enormous costs to society. ${ }^{1-3}$ A cornerstone of treatment uniformly recommended by guidelines on illness management for BD patients is mood-stabilizing medication such as lithium, anticonvulsants, or atypical antipsychotic drugs. ${ }^{1,46}$ Yet, approximately one in two individuals with BD are poorly adherent with prescribed medication treatments, ${ }^{7-10}$ resulting in a variety of negative consequences, including relapse, rehospitalization, suicide, and significantly increased costs of care..$^{3,11-17}$

Although evidence-based interventions to change health behavior exist, their high costs and resource-intensive nature make them impractical for many BD patients. ${ }^{18-20}$
Correspondence: Martha Sajatovic Department of Psychiatry, University Hospitals Case Medical Center, 10524 Euclid Avenue, Seventh Floor, Cleveland, $\mathrm{OH} 44106$, USA

Tel +l 2168442808

Fax +I 4404231863

Email martha.sajatovic@uhhospitals.org 
Technology-assisted approaches are increasingly being studied and used to help people with mental illnesses, such as BD, better self-manage their health. ${ }^{21-23}$ These investigators have been developing a technology-enabled adherence enhancement system for patients with BD. This pilot study evaluated the preliminary feasibility of the system, patient satisfaction with the technology, and effects on medication adherence, knowledge of BD, and BD symptoms. Given the growing importance of technology in illness self-management, practical and patient-friendly approaches are needed.

\section{Methods}

\section{Study overview}

This was a prospective analysis of a multicomponent, technology-assisted adherence enhancement system in five patients with BD, who tested the system over a 15-day period. The DialogMeds-BD system (Figure 1) is designed to address the barriers to adherence in BD patients. The DialogMeds-BD components include: 1) an automated pill cap with remote monitoring sensor; 2) a multimedia adherence enhancement (MAE) program; and 3) a treatment incentive program (TIP) for motivating patients to remain adherent and improve treatment-related knowledge and skills. System data are intended for viewing by clinicians in real-time, web-based format, which can be used to help in treatment planning and medical decision-making. This preliminary study evaluated

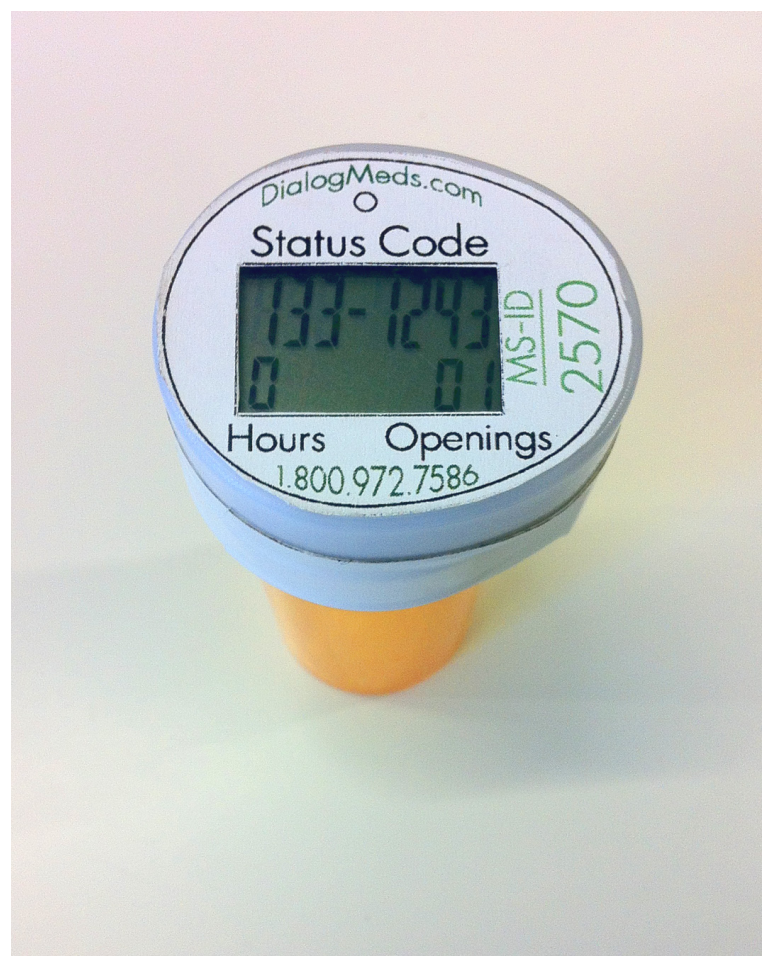

Figure I Illustration of an automated pill cap to monitor pill adherence in patients with bipolar disorder (BD). the feasibility of use, patient satisfaction with the system, and effects on BD adherence, knowledge, and symptoms.

\section{Pill-cap monitoring sensor}

A prototype electronic pill-cap sensor was developed for this project. Designed to be attached to standard medication bottles dispensed by commercial pharmacies, the specialized sensor has an embedded computer chip and a two-line LCD screen. When removed from its bottle, the sensor records the instance of the bottle opening and stores it in memory, thereby making it possible to track patients' presumed dosing episodes over the course of their treatment regimen. To help patients monitor their dosing for a given day, the LCD screen displays the hours since the last cap opening, as well as the number of total openings in the last 24 hours. A detailed adherence record that includes the number of openings per day for the previous 8-day period is also stored in the memory chip in the form of a "status code." A 7-10 digit encrypted version of this status code is also displayed on the LCD screen. The encryption algorithm conceals the adherence data embedded in the status code by performing a logical XOR operation involving a rolling set of codes unique to each cap, resulting in what appears to be a random number. The encrypted code can then be entered by patients during weekly interactive voice response (IVR) check-ins, at which point it is decoded and included in the provider reports. Figure 2 illustrates a sample provider report.

\section{MAE}

The MAE program is designed to educate patients about illness knowledge and self-management skills empirically related to successful clinical outcomes. Materials for the program were adapted from an effective intervention designed to improve treatment adherence attitudes and behaviors among BD patients. ${ }^{24,25}$ Topics were condensed and presented in a series of brief 2-5 minute segments. A narrative script of a sample session is noted in Figure 3.

\section{TIP}

The TIP is a contingency management approach for motivating patients to improve their treatment knowledge and remain adherent to their prescribed regimen. TIP points (TIPS), which can be redeemed for cash, are awarded to patients for completing MAE materials, checking-in as scheduled, and completing assessments.

\section{Participants}

Five $(\mathrm{N}=5)$ individuals with $\mathrm{BD}$ were recruited from the clinical and research practice of the psychiatrist investigator. Inclusion 


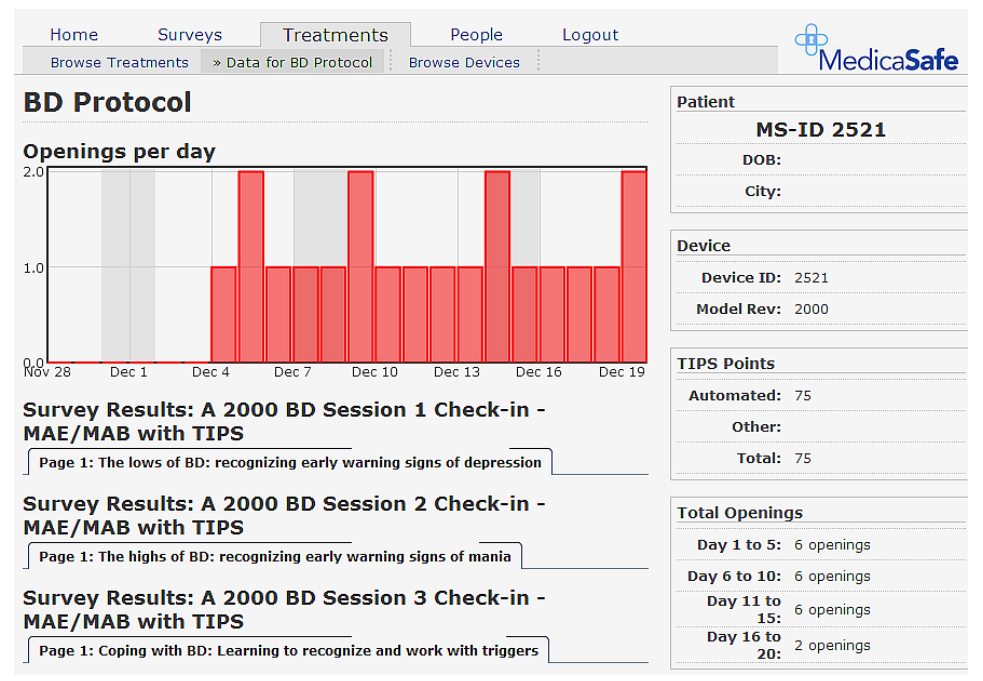

Figure 2 Clinician report provided by a technology-enabled adherence enhancement system for people with bipolar disorder (BD). Abbreviations: $\mathrm{BD}$, bipolar disorder; MAE, multimedia adherence enhancement; TIPS, treatment incentive program points.

\section{Session 1: The lows of BD: recognizing early warning signs of depression:}

[Dr Voice] "Hi, I'm Doctor X. Everyone experiences mood changes in response to every day events. When good things happen, we tend to feel happy; when bad things happen, we tend to feel sad. But when you have bipolar disorder, it's different. Everything is exaggerated. The lows are lower, the highs are higher, and mood swings can affect your functioning."

[Peer Voice] “I'm Jane. When I'm having a depressive episode, I'm really, really down about everything. I'm sad all the time and can't enjoy anything; even the things I usually like the most. I have trouble eating, sleeping, or being productive at all. Sometimes I can't even get out of bed. And the worst part is it feels like it will never get better. And so I think, well, why bother taking my medication anyway. It's not going to work. My doctor tells me I need my meds, but l'm not convinced."

[Dr Voice] "A number of thoughts, feelings, and behaviors are early warning signs of oncoming depression. Early warning sign thoughts include:"

[Assistant voice]" _ The belief that things will not get better,

_Problems with concentration, memory, and decision-making,

_Feeling helpless and hopeless,

- Thoughts about dying or suicide,

- A sense that others are against you,

_Hearing voices or seeing visions,

_Focus on negative things from the past".

[Dr Voice] "Early warning sign feelings include:"

[Assistant voice] "Lack of energy,

_- Increased or decreased appetite,

_ Less interest in activities you used to enjoy,

_ Not feeling good even when good things happen,

_Feeling guilty or sad."

[Dr Voice] "Early warning sign behaviors include:"

[Assistant voice] "_Restlessness and/or pacing,

_Sleeping too little or too much,

— Trouble starting or finishing projects,

_-Isolating yourself from others,

_Stopping/cutting down on work or usual activities,

_ Getting irritable or tearful easily,

_Using drugs or alcohol,

_Not taking your medications."

[Dr Voice] "If you experience early warning signs of depression, the most important thing for you to do is to be aware of what is happening. Talk to your doctor or clinician and speak with supportive family members or friends. If you are having thoughts of suicide or self-harm, tell your doctor and people in your support network right away so that they can help you get the support you need. If you are having problems with your medication, talk with your doctor right away. Changes and adjustments can be made to make things better. Stopping your medication on your own will almost always make things worse."

Figure 3 Narrative content of a sample multimedia adherence enhancement (MAE) session.

Abbreviation: BD, bipolar disorder. 
criteria required that patients be at least 18 years old and have a clinical diagnosis of BD type I or type II. For this initial assessment of the system, individuals who might be expected to have difficulty in following study procedures were excluded.

\section{Specific procedures}

Pill monitoring sensors were fitted onto an existing BD medication bottle. In the event that individuals were on more than one medication to manage their $\mathrm{BD}$, the sensor was used on their most recently started maintenance $\mathrm{BD}$ medication. Upon in-person enrollment, $\mathrm{BD}$ patients registered by phone with the system. A research assistant (RA) walked the patient through the registration process and initial check-in, as well as reviewed the overall system usage. The registration and check-ins included listening to MAE material by phone and entering answers to adherence questions. Patients then checked in remotely on their own by phone on days 5,10 , and 15 . TIP points were awarded for completing each of the four scheduled phone check-ins.

\section{Usability and acceptance}

Immediately after registering with the system, using a 5-point Likert scale, (one worst, five best) participants rated their satisfaction with the system and were asked to provide a brief explanation for their rating. At the conclusion of the study, participants completed an acceptance survey using a 7-point Likert scale (one worst, seven best) and were asked to provide responses to a series of open-ended questions regarding their perceptions of the system.

\section{Clinical and adherence status measurements}

In addition to collecting basic demographic and clinical data at baseline (age, sex, ethnicity, education level, and current medications), adherence, treatment knowledge, BD symptoms, and perceptions of system usability by phone were assessed at baseline and after the 15-day period of system use. Adherence was assessed with the self-reported Morisky questionnaire. ${ }^{26}$ The internal state scale (ISS) measured BD symptoms. ${ }^{27,28} \mathrm{~A}$ 9-question treatment knowledge test (TKT) measured knowledge of concepts and practices known to be associated with improved treatment outcomes for BD patients and which were specifically covered in the MAE.

\section{Data analysis}

Given the primary feasibility focus of the study, the primary assessments were qualitative (eg, did patients accept and use the system?). Descriptive statistics were calculated for secondary outcomes (adherence, knowledge, and BD symptoms).

\section{Results \\ Enrollment and system implementation}

There were three male and two female participants, mean age 62 years (range 49-71 years), 4/5 (80\%) Caucasian, and 4/5 (80\%) single/divorced or widowed. Mean years of education was 13 years (range 12-14 years). The majority resided in private homes with other people. Most participants $(4 / 5,80 \%)$ were on a single medication for treatment of BD. On average, participants had BD for 21 years (range 5-43 years) and were clinically stable, treated BD patients. These pilot study participants were mostly euthymic or mildly depressed. Possible ratings on the ISS for depressive severity range from 0 to 200 with higher scores representing more severe depression. The study sample ISS scores were reflective of their overall clinical stability with a mean of 40 (SD 56).

There were some challenges with system implementation including medication containers that were too large to easily fit the pill sensor, child-proof pill caps that made it difficult to attach sensors, and some individuals who used multiday pill minders rather than pill bottles dispensed by the pharmacy. In these situations, patients used an empty pill bottle to store their medications for the 15-day study duration. Out of five participants, three (60\%) were able to complete use of the system for the full 15-day period. Pill sensors came off of pill caps in two cases due to being dropped $(\mathrm{N}=1)$ or tape that affixed the sensor coming off $(\mathrm{N}=1)$. Individuals who had sensors come off their pill bottles continued to complete phone-in MAE and other assessments.

\section{Usability and acceptability}

Usability scores on the 5-point scale were high overall with means ranging from 4.8 to 5.0 on all usability questions. Endpoint acceptability scores on the 7-point scale were also high with means ranging from 4.8 to 6.3 . When queried regarding what they liked best about the system, the most common response was the simplicity and ease of use, while the most common complaints or least liked components of the system were related to the difficulty with the temporary (tape) method used to affix the pill caps to the bottle. All patients completed all scheduled check-ins and thus earned the maximum eligible TIPS available.

\section{Adherence, BD symptoms, BD knowledge}

Mean scores on the Morisky scale improved from baseline to follow-up (3.20-3.60). Means on all four subscales of the ISS were all in the direction of improvement. Scores on perceived conflict (baseline 60, endpoint 48), activation (baseline 64, endpoint 12), and depression index (baseline 
40, endpoint 10) all decreased, while scores on well-being (baseline 222, endpoint 258) increased. Finally, mean TKT scores increased from baseline to follow-up (14-19.6) with all five participants demonstrating improved treatment knowledge relative to the baseline test administration.

\section{Clinician interface}

As noted in Figure 2, a graphic showing pill bottle openings per day and TIPS readily provided an overview of adherence behavior and BD education.

\section{Discussion}

Given the pervasive and negative effects of poor adherence on people with $\mathrm{BD}$, approaches that improve adherence can improve outcomes and enhance the efficiency of care. Our preliminary experience with a technology-assisted multiple component adherence enhancement system suggests that the system is well accepted, feasible to implement, and has the potential to improve adherence and related health outcomes in people with BD. Somewhat similar to our experience in that it used a mobile device, focused on adherence in patients with $\mathrm{BD}$, and was administered over a relatively short time-frame, Wenze et al evaluated the feasibility and acceptability of an ecological momentary intervention (EMI), delivered via personal digital assistants (PDAs), to improve treatment adherence. ${ }^{21}$ Overall, participants in the Wenze et al study $(\mathrm{N}=14)$ reported satisfaction with the usefulness, timing and burden of sessions, as well as the method of delivery. ${ }^{21}$ Negative aspects of the PDA-delivered approach were mainly in technical and logistic limitations. While our study also had a number of limitations including small sample size, short-term duration, and a temporary method of affixing pill sensors to pill bottles that was sub-optimal, findings may still be helpful in considering next steps for technology-assisted adherence enhancement methods in BD.

Patients with BD in our sample were overall very accepting of the system and considered it easy to use. However, a sensor that is designed to fit on top of a standard pharmacy pill bottle may not be generalizable for use in people who receive large mail-order pill containers (for example, 90-day supplies of medications that are taken multiple times per day) and for people who transfer their medications to multiday pill minders. Our sample of BD patients was mostly taking a single BD medication daily, and may not be broadly representative of most people with $\mathrm{BD}$. Baldessarini et $\mathrm{al}^{29}$ analyzed US national health plan claims data from 2000 to 2004 and found that approximately one-third of patients with $\mathrm{BD}$ are on medication polytherapy. Polytherapy rates might be expected to be even higher in public sector care where people with BD are more ill and more impaired.

While the symptom changes in our BD sample are too small to generalize, clinical outcomes (adherence, BD knowledge, BD symptoms) were possibly improved (and certainly not worse). It is possible that if the study had been continued for a longer period, these seeming improvements would not have persisted. Depp et $\mathrm{al}^{23}$ very recently reported on a randomized single-blind controlled trial with 82 individuals with BD who completed a four-session psychoeducational intervention and were assigned to 10 weeks of either: 1) mobile device delivered interactive intervention linking patient-reported mood states with personalized self-management strategies, or 2) paper-and-pencil mood monitoring. Participants were assessed at baseline, 6, 12, and 24 weeks follow-up. Compared to the paper-and-pencil condition, participants in the augmented mobile intervention condition had greater reductions in depressive symptoms at 6 and 12 weeks although this was not maintained at 24 weeks. ${ }^{23}$ It is possible that technology-assisted self-management support needs to be continued indefinitely to continue to provide support. On the positive side, technology approaches may be less invasive and expensive than the in-person methods that are used in typical clinical settings. Longer, more rigorous studies are needed to assess the effects of technology-assisted BD self-management on extended health outcomes.

The TIP approach to incentivize patients appears effective, although how this would be operationalized in real-world settings needs further consideration. Examples of widely used health care incentives include health insurance plans that give patients discounts or financial compensation for healthy behaviors like smoking cessation or blood pressure testing. In our multiple component adherence enhancement system, a clinician read-out provides adherence data that is potentially useful for care monitoring and planning. Based in a secure, web-based platform, adherence behavior information can help clinicians to make such key decisions as determination of reasons for sub-optimal medication response (poor adherence vs treatment refractory illness) and changes in drug formulation (consideration of once daily vs multiple times daily dosing vs long-acting or injectable long-acting drugs).

\section{Conclusion}

In conclusion, poor adherence is a major obstacle to good health outcomes for people with BD. Technology-assisted approaches need to be refined that can incentivize adherence, teach patients necessary information and skills to maximize adherence, and inform clinicians on patient adherence patterns. 


\section{Acknowledgment}

This study was supported by an investigator-initiated grant from MedicaSafe, Inc., to the study PI (Sajatovic).

\section{Disclosure}

Martha Sajatovic is a consultant for Prophase, Otsuka, Sunovion, Pfizer, Amgen, and Bracket, and has received grant support from Pfizer, Merck, Ortho-McNeil Janssen, and Janssen. Michael Davis is employed by MedicaSafe Inc. Joseph Nestor is a former employee of MedicaSafe Inc. The other authors report no conflicts of interest in this work.

\section{References}

1. American Psychiatric Association. Practice guideline for the treatment of patients with bipolar disorder (revision). Am J Psychiatry. 2002; 159(4 suppl):1-50.

2. Murray CJ, Lopez AD. Global mortality, disability, and the contribution of risk factors: global burden of disease study. Lancet. 1997; 349(9063):1436-1442.

3. Begley CE, Annegers JF, Swann AC, et al. The lifetime cost of bipolar disorder in the US: an estimate for new cases in 1998. Pharmacoeconomics. 2001;19(5 pt 1):483-495.

4. Keck PE Jr, Perlis RH, Otto MW, Carpenter D, Ross R, Docherty JP. The Expert Consensus Guidelines: Treatment of Bipolar Disorder; 2004. Postgraduate Medicine Report: Special Report: 1-120.

5. Goodwin GM, Young AH. The British association for psychopharmacology guidelines for treatment of bipolar disorder: a summary. J Psychopharmacol. 2003;17(4 suppl):3-6.

6. Yatham LN, Kennedy SH, O'Donovan C, et al. Canadian network for mood and anxiety treatments (CANMAT) guidelines for the management of patients with bipolar disorder: consensus and controversies. Bipolar Disord. 2005;7(suppl 3):5-69.

7. Sajatovic M, Valenstein M, Blow FC, Ganoczy D, Ignacio RV. Treatment adherence with antipsychotic medications in bipolar disorder. Bipolar Disord. 2006;8(3):232-241.

8. Sajatovic M, Valenstein M, Blow F, Ganoczy D, Ignacio RV. Treatment adherence with lithium and anticonvulsant medications among patients with bipolar disorder. Psychiatr Serv. 2007;58(6):855-863.

9. Lingam R, Scott J. Treatment non-adherence in affective disorders. Acta Psychiatr Scand. 2002;105(3):164-172.

10. Perlick DA, Rosenheck RA, Kaczynski R, Kozma L. Medication nonadherence in bipolar disorder: a patient-centered review of research findings. Clin Approaches in Bipolar Disord. 2004;3(2):56-64.

11. Volpp KG, Gurmankin Levy A, Asch DA, et al. A randomized controlled trial of financial incentives for smoking cessation. Cancer Epidemiol Biomarkers Prevent. 2006;15(1):12-18.

12. Griffith JD, Rowan-Szal GA, Roark RR, Simpson DD. Contingency management in outpatient methadone treatment: a meta-analysis. Drug Alcohol Depend. 2000;58:55-66.

13. Gine X, Karlan D, Zinman J. Put Your Money Where Your Butt is: A Commitment Savings Account for Smoking Cessation. Innovations for Poverty Action [Internet]; 2008 [cited March 1 2014]. Available from: http://documents.apec.umn.edu/DKarlanT\&DSemSp08.pdf

Patient Preference and Adherence

\section{Publish your work in this journal}

Patient Preference and Adherence is an international, peer-reviewed, open access journal that focuses on the growing importance of patient preference and adherence throughout the therapeutic continuum. Patient satisfaction, acceptability, quality of life, compliance, persistence and their role in developing new therapeutic modalities and compounds to optimize
14. Forness SR, Kavale KA, Blum IM, Llyod JW. Mage-analysis of metaanalysis: what works in special education and related services? Teach Except Child. 1997;29:4-9.

15. Schumacher JE, Milby JB, Wallace D, et al. Meta-analysis of day treatment and contingency-management dismantling research: Birmingham homeless cocaine studies (1990-2006). J Consult Clin Psychol. 2007; 75(5):823-828.

16. Lussier JP, Heil SH, Mongeon JA, Badger GJ, Higgins ST. A metaanalysis of voucher-based reinforcement therapy for substance use disorders. Addiction. 2006;101(2):192-203.

17. Dutra L, Stathopoulou G, Basden SL, Leyro TM, Powers MB, Otto MW. A meta-analytic review of psychosocial interventions for substance use disorders. Am J Psychiatry. 2008;165(2):179-187.

18. Hulleman CS, Cordray DS. Moving from the lab to the field: the role of fidelity and achieved relative intervention strength. J Res Educ Eff. 2009;2:88-110.

19. Miklowitz DJ, Otto MW, Frank E, et al. Psychosocial treatments for bipolar depression: a 1-year randomized trial from the systematic treatment enhancement program. Arch Gen Psychiatry. 2007;64(4): 419-426.

20. Sauro J, Kindlund E. Using a single usability metric (SUM) to compare the usability of competing products. Paper presented at: Human Computer Interaction International Conference; July 22-27; 2005; Las Vegas, USA.

21. Wenze SJ, Armey MF, Miller IW. Feasibility and acceptability of a mobile intervention to improve treatment adherence in bipolar disorder: a pilot study. Behav Modif. 2014;38(4):497-515.

22. Burns MN, Begale M, Duffecy J, et al. Harnessing context sensing to develop a mobile intervention for depression. JMed Internet Res. 2011; 13(3): 55 .

23. Depp CA, Ceglowski J, Wang VC, et al. Augmenting psychoeducation with a mobile intervention for bipolar disorder: a randomized controlled trial. J Affect Disord. 2015;174:23-30.

24. Sajatovic M, Levin J, Tatsuoka C, et al. Six-month outcomes of customized adherence enhancement (CAE) therapy in bipolar disorder. Bipolar Disord. 2012;14(3):291-300.

25. Sajatovic M, Levin J, Tatsuoka C, et al. Customized adherence enhancement for individuals with bipolar disorder receiving antipsychotic therapy. Psychiatr Serv. 2012;63(2):176-178.

26. Morisky DE, Green LW, Levine DM. Concurrent and predictive validity of a self-reported measure of medication adherence. Med Care. 1986; 24:67-74.

27. Bauer MS, Vojta C, Kinosian B, Altshuler L, Glick H. The internal state scale: replication of its discriminating abilities in a multisite, public sector sample. Bipolar Disord. 2000;2(4):340-346.

28. Bauer MS, Crits-Christoph P, Ball WA, et al. Independent assessment of manic and depressive symptoms by self-rating. Scale characteristics and implications for the study of mania. Arch Gen Psychiatry. 1991;48(9):807-812.

29. Baldessarini R, Henk H, Sklar A, Chang J, Leahy L. Psychotropic medications for patients with bipolar disorder in the United States: polytherapy and adherence. Psychiatr Serv. 2008;59(10):1175-1183. clinical outcomes for existing disease states are major areas of interest for the journal. This journal has been accepted for indexing on PubMed Central. The manuscript management system is completely online and includes a very quick and fair peer-review system, which is all easy to use. Visit http://www. ovepress.com/testimonials.php to read real quotes from published authors. 